\title{
Power Allocation for Balancing Spectrum Efficiency and Power Consumption in Cognitive Relay Networks
}

\author{
Lun Tang, Qing Li, Qian-Bin Chen \\ Chongqing Key Lab of Mobile Communication Technology, Chongqing University of Posts and \\ Telecommunications, Chongqing China
}

\begin{abstract}
In order to guarantee the QoS requirement of secondary users and not to affect the outage probability of primary user in cognitive relay networks, we propose two optimal power allocation models: (1) maximizing the transmission rate of secondary users; (2) minimizing the total power consumption. Theory analysis shows that two optimal power allocation models conflict between spectrum efficiency and power consumption. Furthermore, an optimal power allocation model which joints the transmission rate and the total power consumption in cognitive relay networks is proposed. By using the Lagrangian method, the optimization algorithm for this model is designed. The proposed algorithm can achieve the trade-off between the transmission rate and the total power consumption by varying the weight. Simulation results show that the proposed algorithm can effectively adjust the transmission rate and the total power consumption of secondary users.
\end{abstract}

Index Terms: Cgnitive relay networks; spectrum efficiency; power consumption; power allocation

(C) 2011 Published by MECS Publisher. Selection and/or peer review under responsibility of the Research Association of Modern Education and Computer Science

\section{Introduction}

In cognitive radio networks, secondary user is allowed to gain access to primary user's idle spectrum to improve the spectrum utilization of the licensed spectrum. The concept of cognitive radio has recently attracted considerable interest in wireless communication. Relay cooperative technology has recently emerged as a powerful spatial diversity technique which can improve the spectrum efficiency and the communication reliability. These two techniques can improve the spectrum utilization and the transmission rate. Therefore, the study of cognitive relay which combines cognitive radio with cooperative communication has been recently attracted wide attention. In [1], the effective capacity of cognitive relay networks is investigated under the quality of service (QoS) constraints of secondary users. In [2], the author investigates power control and

* Corresponding author.

E-mail address: tangl@cqupt.edu.cn, qingziq@qq.com, chenqb@cqupt.edu.cn 
proposes the corresponding geometric programming in cooperative cognitive radio networks. In [3], the author analyzes the system performance in terms of the outage capacity and the average capacity. In [4], the power allocation scheme for multi-hop cognitive radio system is studied, and the model of minimizing the total power consumption of relays is proposed. In [5], the author investigates the affect of the minimum outage probability by employing the optimal power control strategy at relay nodes in cognitive relay networks. The above literatures do not research the problem of combining transmission rate with total power consumption of secondary users in cognitive relay networks, not investigate the network performance from the perspective of maximum arrival rate of cognitive link, and not explicitly propose the joint optimization problem of the maximum transmission rate and the minimum transmit power of cognitive relay networks in the optimization objective. In this paper, in order to guarantee the QoS requirements of secondary users and not to affect the outage probability of primary user in cognitive relay networks, we propose an optimal power allocation optimization model of combining transmission rate maximum with total power consumption minimum, and use the Lagrangian method to solve the corresponding optimization problem.

\section{System Model}

Consider a cognitive relay system with the coexistence of primary and secondary networks, as depicted in Fig.1. In this paper, we consider a primary transmit (PU-Tx)-receive (PU-Rx) pair, and a cognitive network which consists of secondary transmitter (SU-Rx), a cognitive relay (R) and a secondary receiver (SU-Tx). Cognitive relay networks adopt two-hop relay model to communicate with each other.

In this paper, we assume that the transmit power of primary user is $P_{P}$, the transmit power of secondary source and cognitive relay is $P_{S}$ and $P_{R}$, respectively. We further assume that the channel coefficient of primary user is denoted by $h_{P}$, the channel coefficients between node $i$ and $j$ are denoted by $h_{i, j}$, where $i \in\{S U-\mathrm{T} x, R, P U-\mathrm{T} x\}$ and $j \in\{S U-\mathrm{R} x, R, P U-\mathrm{R} x\}$. We assume that the power gains are denoted by $^{\gamma_{i, j}}=\left|h_{i, j}\right|^{2}$, where $\gamma_{i, j}$ are independent and subject to exponentially distributed with parameter $\sigma_{i, j}$.

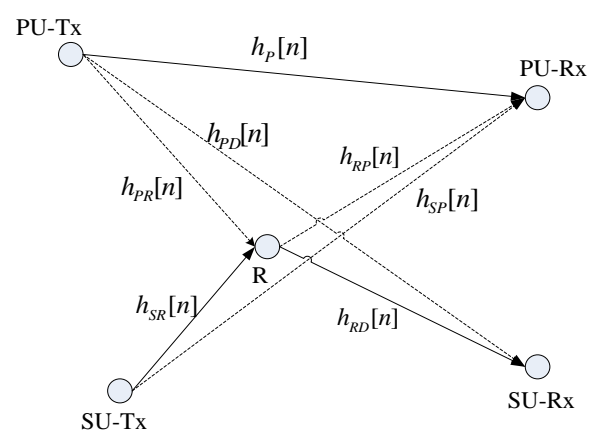

Figure 1. System model for cognitive relay network

We assume that the secondary user's transmission is transparent to primary user; the primary transmitter uses fixed power $P_{P}$ to guarantee its minimum transmission rate $R_{P}$. Thus, the secondary transmitter and the cognitive relay can adjust their transmit power $P_{S}$ and $P_{R}$ with the range of $P_{\min } \leq P_{S} \leq P_{\max }$ and $P_{\min } \leq P_{R} \leq P_{\max }$ to fulfill the two basic goals listed as follows: 
(1) Selfish-goal: On the premise of guaranteeing the outage probability of primary user, maximizing the transmission rate of secondary users and trying to transmit as much information for it-self as possible.

(2) Energy-efficiency-goal: Under the QoS requirement of secondary users, minimizing the total transmit power to reduce energy consumption.

According to the Shannon, at a certain time $\mathrm{n}$, the data transmission rate of secondary user in two-hop model can be written as

$$
R[n]=B \frac{1}{2} \min \left\{\log _{2}\left(1+\frac{P_{S}\left|h_{S R}\right|^{2}}{P_{P}\left|h_{P R}\right|^{2}+N_{0} B}\right), \log _{2}\left(1+\frac{P_{\mathrm{R}}\left|h_{\mathrm{RD}}\right|^{2}}{P_{P}\left|h_{\mathrm{PD}}\right|^{2}+N_{0} B}\right)\right\}
$$

where $N_{0}$ denotes noise power, and subjects to the complex Gaussian distribution with zero-mean.

\section{Mathemathematical Model of Optimization Problem}

On the premise of guaranteeing the outage probability of primary user and the QoS requirement of secondary users, we can use the transmission rate to measure the performance of cognitive relay networks. If the optimization goal is to maximize the achieved rate of the whole networks, we should solve the following optimization problem:

$$
\begin{array}{ll} 
& \max _{\left\{P_{S}, P_{R}\right\}} R[n] \\
\text { s.t. } & P_{\mathrm{r}}\left(R[n] \leq R_{\min }\right) \leq P_{t \text { arg } e t}^{\text {out }} \\
& P_{S}\left|h_{S P}\right|^{2} \leq I_{t h} \\
& P_{R}\left|h_{R P}\right|^{2} \leq I_{t h} \\
& P_{\min } \leq P_{S} \leq P_{\max } \\
& P_{\min } \leq P_{R} \leq P_{\max }
\end{array}
$$

where constraint (2a) satisfy the QoS requirement of secondary users while constraints (2b) and (2c) guarantee the protection of primary user, in which $P_{t \text { arget }}^{\text {out }}$ is the target outage probability and $I_{\text {th }}$ denotes the maximum tolerable interference introduced to primary user. ${ }^{R_{\min }}$ denotes the minimum transmission rate which satisfies the QoS requirement of secondary user. $P_{\max }$ and $P_{\min }$ denote the maximum and minimum transmit power, respectively.

Besides, from the perspective of green communication, all links should reduce the power consumption to save energy:

$$
\begin{array}{ll}
\min _{\left\{P_{S}, P_{R}\right\}} & P=P_{S}+P_{R} \\
\text { s.t } & (2 \mathrm{a})-(2 e)
\end{array}
$$

As can be seen from the above two optimization model, the transmission rate and the total power consumption of secondary users compose a mathematical programming problem which consists of two goals. The transmission rate and the total power consumption connect by constraints (2a)-(2e). We can solve the problem of two objectives by using the method of weigh-adding combination, the weighted factor is denoted by $\omega$ $(0<\omega<1)$, we can adjust a weight $\omega$ to balance selfish-goal and energy- efficiency-goal. On the premise of 
satisfying the QoS requirement of secondary users and guaranteeing the minimum outage probability of primary user, the double objectives can be transformed into a single objective optimization problem, therefore, the optimization model of the trade-off between transmission rate and total power can be written:

$$
\begin{array}{ll}
\max _{\left\{P_{S}, P_{R}\right\}} & (\omega R[n]-(1-\omega) P) \quad(0<\omega<1) \\
\text { s.t. } & (2 \mathrm{a})-(2 e)
\end{array}
$$

The problem (4) combines the transmission rate maximum with the total power consumption minimum of secondary users. When $\omega=1$, it denotes the maximum transmission rate of secondary users, which can achieve the selfish-goal; when $\omega=0$, it denotes the minimum total transmit power, which can achieve the energyefficiency-goal.

\section{Optimal Power Allcation}

Considering the trade-off of transmission rate and total power, we can use the Lagrangian method to get the optimal solution of the objective function (4) by introducing the Lagrangian multiplier for constraints in (2a)(2c). The corresponding Lagrangian function can be written as

$$
\begin{aligned}
& L\left(\mathrm{P}_{S}, \mathrm{P}_{\mathrm{R}}, \lambda, \mu, v\right)=\omega B \frac{1}{2} \log _{2}\left(1+\frac{P_{S}\left|h_{S R}\right|^{2}}{P_{P} h_{P R}+N_{0} B}\right)-(1-\omega)\left\{P_{S}+\mathrm{P}_{\mathrm{R}}\right\} \\
& +\lambda\left\{\mathrm{P}_{\mathrm{r}}\left(R \leq R_{\min }\right)-P_{\text {target }}^{\text {out }}\right\}+\mu\left(P_{S}\left|h_{S P}\right|^{2}-I_{t h}\right)+\nu\left(P_{R}\left|h_{R P}\right|^{2}-I_{t h}\right)
\end{aligned}
$$

where $\lambda, \mu$ and $v$ are the vectors of Lagrangian multipliers.

For energy-efficient communications, we should allocate the right power to relay node so that

$$
\frac{P_{S}\left|h_{S R}\right|^{2}}{P_{P}\left|h_{P R}\right|^{2}+N_{0} B}=\frac{P_{\mathrm{R}}\left|h_{\mathrm{RD}}\right|^{2}}{P_{P}\left|h_{\mathrm{PD}}\right|^{2}+N_{0} B}
$$

In order to guarantee the QoS requirement of secondary users, substitute (6) into $P_{\mathrm{r}}\left(R[n] \leq R_{\min }\right)$. Then the outage probability of secondary users can be written as

$$
\begin{aligned}
P_{S}^{\text {out }} & =P_{\mathrm{r}}\left(R[n] \leq R_{\min }\right) \\
& =P_{r}\left\{\left|h_{S R}\right|^{2} \leq \frac{\left(2^{2 R_{\min }}-1\right)\left(P_{P}\left|h_{P R}\right|^{2}+N_{0} B\right)}{P_{S}}\right\}
\end{aligned}
$$

Since the power gains $\left|h_{S R}\right|^{2}$ and $\left|h_{P R}\right|^{2}$ subject to exponentially distributed with parameter $\sigma_{i, j}$, the outage probability in (7) can be written as

$$
\begin{aligned}
& P_{S}^{\text {out }}=P_{r}\left\{\left|h_{S R}\right|^{2} \leq \frac{\left(2^{2 R_{\min }}-1\right)\left(P_{P}\left|h_{P R}\right|^{2}+N_{0} B\right)}{P_{S}}\right\} \\
& =1-\frac{P_{S} \sigma_{S R}}{\sigma_{P R}\left(2^{2 R_{\min }}-1\right) P_{P}+P_{S} \sigma_{S R}} \exp \left\{-\frac{\left(2^{2 R_{\min }}-1\right) N_{0} B}{P_{S} \sigma_{S R}}\right\}
\end{aligned}
$$

Combining (5), (6) with (8), we can simplify its Lagrangian expression as: 


$$
\begin{aligned}
& L\left(\mathrm{P}_{S}, \lambda, \mu, v\right)=\omega B \frac{1}{2} \log _{2}\left(1+\frac{P_{S}\left|h_{S R}\right|^{2}}{P_{P} h_{P R}+N_{0} B}\right) \\
& -(1-\omega)\left\{P_{S}+\frac{P_{S} h_{S R}\left(P_{P}\left|h_{P D}\right|^{2}+N_{0} B\right)}{h_{R D}\left(P_{P}\left|h_{P R}\right|^{2}+N_{0} B\right)}\right\} \\
& +\lambda\left\{1-\frac{P_{S} \sigma_{S R}}{\sigma_{P R}\left(2^{2 R_{\min }}-1\right) P_{P}+P_{S} \sigma_{S R}} \exp \left\{-\frac{\left(2^{2 R_{\min }}-1\right) N_{0} B}{P_{S} \sigma_{S R}}\right\}-P_{t a r g e t}^{\text {out }}\right\} \\
& +\mu\left(P_{S}\left|h_{S P}\right|^{2}-I_{t h}\right)+v\left\{\frac{P_{S} h_{S R}\left(P_{P}\left|h_{P D}\right|^{2}+N_{0} B\right)}{h_{R D}\left(P_{P}\left|h_{P R}\right|^{2}+N_{0} B\right)}\left|h_{R P}\right|^{2}-I_{t h}\right\}
\end{aligned}
$$

Then, we use gradient iteration to derive the optimal solution ${ }_{S}^{*}$ :

$P_{S}\left(t_{1}+1\right)=\left[P_{S}\left(t_{1}\right)+\alpha\left(t_{1}\right) f\left(P_{S}\right)\right]_{X}$

where $f\left(P_{S}\right)=\frac{\partial L\left(P_{S}, \lambda, \mu, v\right)}{\partial P_{S}}, t_{1}$ denotes iteration index, as the inner circle, $\alpha\left(t_{1}\right)$ is the appropriate step-size, $[\square]_{X}$ denotes the projection of the feasible set $X \stackrel{\Delta}{=}\left\{P_{S}: P_{\min } \leq P_{S} \leq P_{\max }\right\}$.

By using the sub-gradient projection method, the optimal variables $\lambda^{*}, \mu^{*}, v^{*}$ can be obtained by updating $\lambda, \mu, v$ :

$$
\begin{aligned}
& \lambda(t+1)=[\lambda(t)-\beta(t) f(\lambda)]^{+} \\
& \mu(t+1)=[\mu(t)-\beta(t) f(\mu)]^{+} \\
& v(t+1)=[v(t)-\beta(t) f(v)]^{+}
\end{aligned}
$$

where

$$
\begin{aligned}
& f(\lambda)=1-P_{\text {target }}^{\text {out }}-\frac{P_{S}^{*} \sigma_{S R}}{\sigma_{P R}\left(2^{2 R_{\min }}-1\right) P_{P}+P_{S}^{*} \sigma_{S R}} \exp \left\{-\frac{\left(2^{2 R_{\min }}-1\right) N_{0} B}{P_{S}^{*} \sigma_{S R}}\right\} \\
& f(\mu)=P_{S}^{*}\left|h_{S P}\right|^{2}-I_{\text {th }} \\
& f(v)=\frac{P_{S}^{*}\left|h_{S R}\right|^{2}\left(P_{P}\left|h_{P \mathrm{D}}\right|^{2}+N_{0} B\right)}{\left|h_{R D}\right|^{2}\left(P_{P}\left|h_{P R}\right|^{2}+N_{0} B\right)}\left|h_{R P}\right|^{2}-I_{t h}
\end{aligned}
$$

$[\square]^{+}$denotes the objection of the positive real number set, $t$ denotes iteration index, as the outer circle, $\beta(t)$ is the appropriate step-size in iteration $t$ which satisfies

$$
\sum_{t=1}^{\infty} \beta(t)=\infty, \quad \lim _{t \rightarrow \infty} \beta(t)=0
$$

Finally, the optimal initial variable $P_{S}^{*}$ can be recovered via the optimal variables $\lambda^{*}, \mu^{*}, v^{*}$. 


\section{Simunation Results}

In this paper, we validate the efficiency of the proposed algorithm by MATLAB simulation platform. The channel gains can be expressed as $\gamma_{i, j}=\left|h_{i, j}\right|^{2}=d_{i, j}^{-\alpha}$, where $d_{i, j}$ is the distance between $i$ and ${ }^{j}$, and $\alpha$ is path fading coefficient. In the simulation, we consider the cognitive relay networks placed in a $5 m \times 5 m$ square area, in which $d_{p \mathrm{~d}}=d_{s p}=d_{p r}=d_{r p}=2 m$ and $d_{r d}=d_{s r}=0.6 m$. Besides, the mean of the channel gain is $\sigma_{i, j}=\left(d_{i, j}\right)^{-\alpha}$, in which the path-loss factor is $\alpha=4$. The specific parameters used in this simulation are: $N_{0} B=0 d B, P_{\min }=0 d B, P_{\max }=15 d B$ and $I_{t h}=0 d B$, respectively.

\section{A. Maximizing the transmission rate of secondary users}

Figure 2 shows the convergence of algorithm of the objective function $R[n]$. After the optimization algorithm iterate 200 times, the objective function $R[n]$ converges to the optimal solution is about $2.97 \mathrm{bit} / \mathrm{s}$, meanwhile the total power consumption of cognitive system is about $14.4 \mathrm{~dB}$.
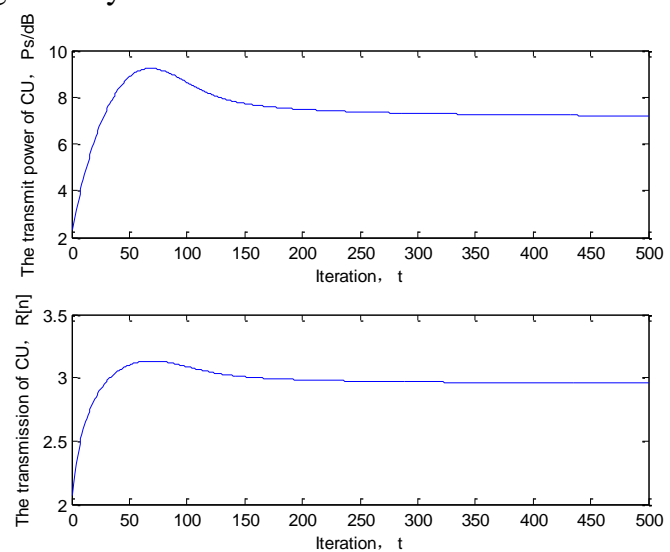

Figure 2. Transmit power (up) and Transmission rate (down) of secondary user for cognitive relay networks $(\omega=1)$

\section{B. Minimizing the total power consumption of secondary users}

Figure 3 shows the convergence of algorithm of the objective function $P=P_{S}+P_{\mathrm{R}}$. After the optimization algorithm iterate 400 times, the total power converges to the optimal solution is about 3.6dB. Compared with A, we save the total transmit power $10.8 \mathrm{~dB}$ to achieve energy-saving goal of cognitive relay networks. 

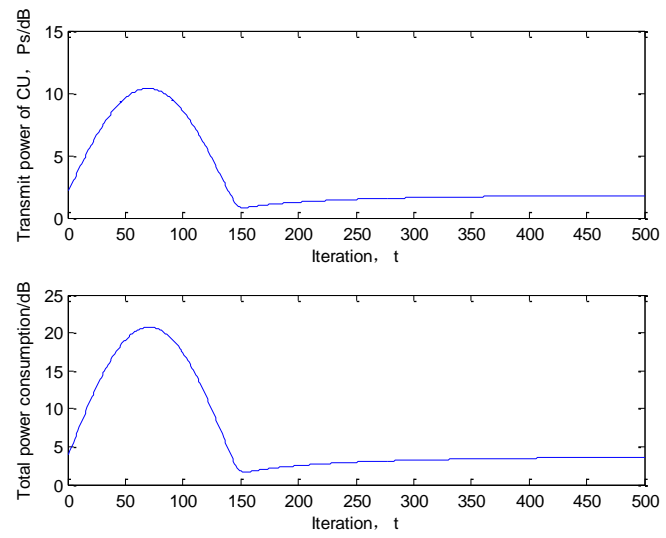

Figure 3. Total power consumption of secondary user for cognitive relay networks $(\omega=0)$

\section{The trade-off between the rate and the total power}

Fig.4 depicts the relationship between the weight $\omega$ and the objective function (4). In the figure, we can find that the solution of the objective function (4) can achieve maximum value when $\omega$ is about 0.1 , at present, the total power consumption of cognitive system is about $9 \mathrm{~dB}$. Compared with $\mathrm{A}$, it saves the power by $5.4 \mathrm{~dB}$, while compared with $\mathrm{B}$, the consumed power increases by $5.4 \mathrm{~dB}$. That is, in practice, under the constraint of the outage probability of primary user, we can still achieve the goal of reducing the total power consumption and interference by reducing the total transmit power of secondary users. At the same time, the QoS requirement of secondary users also can be satisfied.

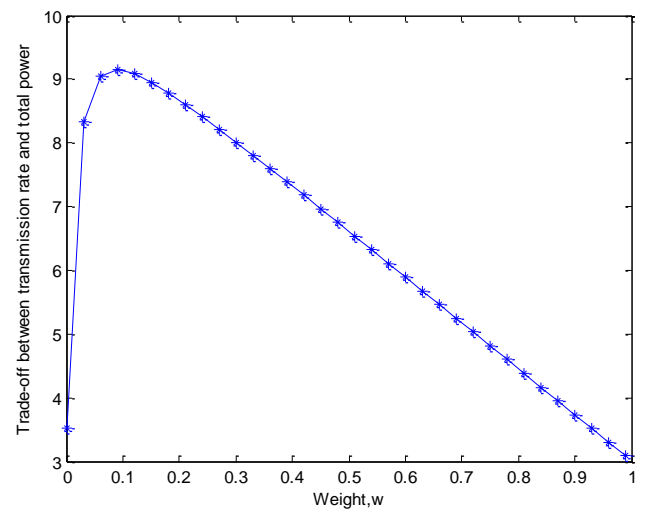

Figure 4. Weight $\omega$ vs. Trade-off between the rate and the total power for cognitive relay networks

\section{Conclusion}

In this paper, on the premise of guaranteeing the QoS requirement of secondary user and the outage probability of primary user, we investigate two aspects of secondary users: (1) maximizing the transmission rate; (2) minimizing the total power consumption. Based on the above analysis, we propose a joint rate and power control optimization model for cognitive relay networks, and we further propose the corresponding power 
control algorithm. Simulation results show the availability of the proposed algorithm. We can obtain a compromise between the transmission rate and the total power consumption of secondary users by adjusting weight $\omega$ and achieve a trade-off between selfish-goal and energy-efficiency-goal in cognitive relay networks.

\section{Acknowledgment}

This work was supported by the National Key Technology R\&D Program (2008BAH30B10), the National Science and Technology Major Special Project of China (2008ZX03003-005), the Natural Science Foundation of China (60972070), the Natural Science Foundation of Chongqing, China (CSTC2009BA2090) and the Foundation of Chongqing Educational Committee, China (KJ090501, KJ100514).

\section{References}

[1] Leila Musavian, Sonia Aissa, "Cross-layer Analysis of Cognitive Radio Relay Networks under Quality of Service Constraints," Vehicular Technology Conference,2009.VTC Spring 2009. IEEE 69th.

[2] Dong Li, Xianua Dai, "Power Control in Cooperative Cognitive Radio Networks by Geometric Programming," Proceedings of the 15th Asia-Pacific Conference on Communication (APCC 2009)-027.

[3] Hongbing Cheng, Member, Yu-Dong Yao, "Cognitive-Ralay Based Inter-Cell Interference Cancelation in Cellular Systems,” IEEE Trans. Vehicular Technology, Forthcoming, pp.1-9, 2010.

[4] Jan Mietzner, Lutz Lampe, Robert Schober, "Distributed Transmit Power Allocation for Multihop Cognitive-Radio Systems, ” IEEE Trans. Wirel. Commun. vol. 8, no. 10, pp.5187-5201, October. 2009.

[5] Wenjing Yue, Baoyu Zheng, Qingmin Meng. "Optimal Power Control for Cognitive Relay Networks over Fading Channels," Second International Conference on Future Networks. pp.281-285, March. 2010. 